\title{
Pre-treatment for exposure to organophosphate cholinesterase inhibitors
}

\author{
Georg Petroianu', Syed M Nurulain ${ }^{2,3}$, Mohamed Y Hassan², Kamil Kuca ${ }^{4}$, Dietrich E Lorke ${ }^{1}$ \\ ${ }^{I}$ Florida International University, USA, ${ }^{2}$ College of Medicine \& Health Sciences, UAE University, UAE, ${ }^{3}$ Institute of \\ Information Technology, Islamabad, Pakistan, ${ }^{4}$ University of Hradec Kralove, Czech Republic
}

Introduction: Organophosphates (OPs), useful agents as pesticides, also represent a serious health hazard due to acetylcholine-esterase (AChE) inhibition. Standard therapy with atropine and established oxime-type enzyme reactivators is unsatisfactory. Better therapeutic results are obtained when reversible AChE inhibitors are administered before OP exposure. In search of a compound that is efficacious prophylactically in the exposure to a broad range of OPs, we have compared the prophylactic efficacy of 10 known AChE inhibitors, either already used clinically (physostigmine, pyridostigmine, ranitidine, tiapride, tacrine, amiloride, metoclopramide, methylene blue) or developed for future therapeutic use (7-methoxytacrine, K-27).

Methods: AChE-inhibitory activity of the AChE-inhibitors was quantified by determining their IC50. The relative risk of death (RR) was estimated by Cox survival analysis in rats that were first administered the reversible AChE-inhibitor at equitoxic dosage, followed by an OP: diisopropylfluorophosphate (DFP), paraoxon-methyl, paraoxon-ethyl, terbufossulfone, azinphos or dicotophos, with $\mathrm{RR}=1$ for animals receiving no pretreatment.

Results: Physostigmine was the strongest in vitro AChE-inhibitor $>7$-methoxytacrine $>$ tacrine $>$ pyridostigmine $>$ methylene-blue $>$ ranitidine $>$ metoclopramide $>$ amiloride $>$ tiapride $>\mathrm{K}-27$.

The following RRs were observed for the tested reversible AChE-inhibitors administered before the 6 different OPs:

The best RR-reducing efficacy (lowest overall RR value) was observed for K-27 and physostigmine. No correlation was observed between the IC50 values of the reversible AChE-inhibitors and their protective efficacy.

Conclusions: K-27 can be considered an efficacious broad-spectrum prophylactic agent in case of imminent organophosphate exposure. This may be related to its AChE reactivating activity rather than its AChE-inhibition. Given its poor penetration into the brain, $\mathrm{K}-27$ may be considered a promising pretreatment agent. 